\title{
Nordhaus-Gaddum Type Results for Total Domination
}

\author{
Michael A. Henning $\|^{\dagger}$ \\ Ernst J. Joubert| \\ Department of Mathematics, University of Johannesburg, South Africa \\ received $30^{\text {th }}$ March 2011, accepted $3^{\text {rd }}$ December 2011.
}

Justin Southey

\begin{abstract}
A Nordhaus-Gaddum-type result is a (tight) lower or upper bound on the sum or product of a parameter of a graph and its complement. In this paper we study Nordhaus-Gaddum-type results for total domination. We examine the sum and product of $\gamma_{t}\left(G_{1}\right)$ and $\gamma_{t}\left(G_{2}\right)$ where $G_{1} \oplus G_{2}=K(s, s)$, and $\gamma_{t}$ is the total domination number. We show that the maximum value of the sum of the total domination numbers of $G_{1}$ and $G_{2}$ is $2 s+4$, with equality if and only if $G_{1}=s K_{2}$ or $G_{2}=s K_{2}$, while the maximum value of the product of the total domination numbers of $G_{1}$ and $G_{2}$ is $\max \left\{8 s,\left\lfloor(s+6)^{2} / 4\right\rfloor\right\}$.
\end{abstract}

Keywords: Nordhaus-Gaddum; Total domination; Relative complement

\section{Introduction}

In this paper, we continue the study of total domination in graphs which was introduced by Cockayne, Dawes, and Hedetniemi Cockayne et al. (1980). A total dominating set, abbreviated TDS, of a graph $G$ is a set $S$ of vertices of $G$ such that every vertex is adjacent to a vertex in $S$. Every graph without isolated vertices has a TDS, since $S=V(G)$ is such a set. The total domination number of $G$, denoted by $\gamma_{t}(G)$, is the minimum cardinality of a TDS. A TDS of $G$ of cardinality $\gamma_{t}(G)$ is called a $\gamma_{t}(G)$-set. Total domination in graphs is now well studied in graph theory. The literature on this subject has been surveyed and detailed in the two books by Haynes, Hedetniemi, and Slater Haynes et al. (1998a b). A recent survey of total domination in graphs can be found in Henning (2009).

In 1956 the original paper Nordhaus and Gaddum (1956) by Nordhaus and Gaddum appeared. In it they gave sharp bounds on the sum and product of the chromatic numbers of a graph and its complement. Since then such results have been given for several parameters; see, for example, Chartrand and Mitchem (1971); Füredi et al. (2005). Nordhaus-Gaddum inequalities involving domination parameters in graphs have been studied in several papers; see, for example, Arumugan and Thuraiswamy (1996); Cockayne et al. (1980); Erfang et al. (2004); Favaron et al. (2010); Goddard and Henning (2003); Goddard et al.

\footnotetext{
†Email: mahenning@uj.ac.za

¥Email: ejoubert@uj.ac.za

Email: just.so@presentable.me
}

1365-8050 (c) 2011 Discrete Mathematics and Theoretical Computer Science (DMTCS), Nancy, France 
(1992); Harary and Haynes (1996); Hattingh et al. (2008); Jaeger and Payan (1972); Joseph and Arumugam (1995); Payan and Xuong (1982) and elsewhere. In particular, we remark that in the introductory paper on total domination, Cockayne, Dawes, and Hedetniemi Cockayne et al. (1980) proved a NordhausGaddum bound for the sum of the total domination numbers of a graph and its complement, while the authors Henning et al. (2011) gave a bound for their product.

Theorem 1 (Cockayne et al.(1980); Henning et al. (2011)) Let $G$ be a graph of order $n$ such that neither $G$ nor $\bar{G}$ contains isolated vertices. Then the following holds.

(a) $\gamma_{t}(G)+\gamma_{t}(\bar{G}) \leq n+2$.

(b) $\gamma_{t}(G) \gamma_{t}(\bar{G}) \leq 2 n$.

In both inequalities, equality holds if and only if $G$ or $\bar{G}$ consists of disjoint copies of $K_{2}$.

For an overview of Nordhaus-Gaddum inequalities for domination-related parameters we refer the reader to Chapter 10 in the domination book by Haynes, Hedetniemi, and Slater Haynes et al. (1998a).

Plesník $\mathrm{k}$ (1978) was the first to extend Nordhaus and Gaddum's results to the case where the complete graph is factored into several factors. Goddard, Henning and Swart Goddard et al. (1992) continued this approach and considered the domination number and $G_{1} \oplus G_{2} \oplus G_{3}=K_{n}$. They also looked at another variation on Nordhaus-Gaddum type results in which they extended the concept by considering $G_{1} \oplus G_{2}=K(s, s)$ rather than $G_{1} \oplus G_{2}=K_{n}$. (If $G$ and $H$ are graphs on the same vertex set but with disjoint edge sets, then $G \oplus H$ denotes the graph whose edge set is the union of their edge sets.)

In this paper, we focus our attention on Nordhaus-Gaddum type results for the total domination number. In particular, we establish upper bounds on the sums and products of the total domination numbers of $G_{1}$ and $G_{2}$, where $G_{1} \oplus G_{2}=K(s, s)$, and neither $G_{1}$ nor $G_{2}$ contains isolated vertices.

\subsection{Notation}

For notation and graph theory terminology we in general follow Haynes et al. (1998a). Specifically, let $G=(V, E)$ be a graph with vertex set $V$ of order $n=|V|$ and edge set $E$ of size $m=|E|$, and let $v$ be a vertex in $V$. The open neighborhood of $v$ is $N(v)=\{u \in V \mid u v \in E\}$ and the closed neighborhood of $v$ is $N[v]=\{v\} \cup N(v)$. For a set $S \subseteq V$, its open neighborhood is the set $N(S)=\cup_{v \in S} N(v)$ and its closed neighborhood is the set $N[S]=N(S) \cup S$. For a set $S \subseteq V$, the subgraph induced by $S$ is denoted by $G[S]$. For subsets $S, T \subseteq V$, the set $S$ totally dominates the set $T$ if $T \subseteq N(S)$, while $S$ dominates $T$ if $T \subseteq N[S]$. In particular, if $S$ dominates $V$, then $S$ is called a dominating set in $G$. The minimum degree among the vertices of $G$ is denoted by $\delta(G)$. A cycle on $n$ vertices is denoted by $C_{n}$ and a path on $n$ vertices by $P_{n}$, while a complete graph on $n$ vertices is denoted by $K_{n}$. If $G$ is a disjoint union of $k$ copies of a graph $F$, we write $G=k F$. For disjoint subsets $X$ and $Y$ of $V$, we let $G[X, Y]$ denote the set of all edges of $G$ between $X$ and $Y$.

\section{Total Domination Number and Relative Complement}

If $G$ is a subgraph of $H$, then the graph $H-E(G)$ is called the complement of $G$ relative to $H$. In Goddard et al. (1992), the authors determined the graphs $H$ with respect to which complements are always unique in the following sense: if $G_{1}$ and $G_{2}$ are isomorphic subgraphs of $H$, then their complements $H-E\left(G_{1}\right)$ and $H-E\left(G_{2}\right)$ are isomorphic. 
Theorem 2 (Goddard et al. (1992)) Let $H$ be a graph without isolated vertices with respect to which complements are always unique. Then $H$ is one of the following: (a) $r K(1, s),(b) r K_{3}$, (c) $K_{s}$, (d) $C_{5}$, or $(e) K(s, s)$, for some integers $r$ and/or $s$.

The above results suggest that the complete bipartite graph $K(s, s)$ is an obvious alternate to $K_{n}$ in Nordhaus-Gaddum results. In Goddard et al. (1992), the sums and products of $\psi\left(G_{1}\right)$ and $\psi\left(G_{2}\right)$ are examined where $G_{1} \oplus G_{2}=K(s, s)$, and $\psi$ is the independence, domination, or independent domination number, inter alia. In this section we take $\psi$ to be the total domination number.

\subsection{Notation}

For the rest of the section we shall assume that $K(s, s)$ has partite sets $\mathcal{L}$ and $\mathcal{R}$ (standing for "left" and "right"), and that $G_{1} \oplus G_{2}=K(s, s)$ where neither $G_{1}$ nor $G_{2}$ have an isolated vertex. For $G \subset K(s, s)$, let $\delta^{L}(G)$ denote the minimum degree of a vertex of $\mathcal{L}$. Define $\delta^{R}(G), \Delta^{L}(G)$ and $\Delta^{R}(G)$ similarly. Further we shall abbreviate parameters by writing $\gamma_{t}^{i}$ for $\gamma_{t}\left(G_{i}\right), \psi_{i}$ for $\psi\left(G_{i}\right)$ for parameters $\psi \neq \gamma_{t}$, $N_{i}(v)$ for $N_{G_{i}}(v), E_{i}$ for $E\left(G_{i}\right), V_{i}$ for $V\left(G_{i}\right)$, and $m_{i}$ for the size of $G_{i}$, for $i \in\{1,2\}$.

Recall that for subsets $S, T \subseteq V$, the set $S$ totally dominates the set $T$ if $T \subseteq N(S)$. Let $G \subset K(s, s)$. A left total dominating set, abbreviated left TDS, of $G$ is a set of vertices that totally dominates $\mathcal{L}$; that is, a left TDS is a set $S$ of vertices of $G$ such that $\mathcal{L} \subseteq N(S)$. Necessarily a left TDS of $G$ belongs to

$\mathcal{R}$. The minimum cardinality of a left TDS in $G$ is called the left domination number, denoted $\ell(G)$, of $G$. Similarly, a right total dominating set, abbreviated right TDS, of $G$ is a set of vertices that totally dominates $\mathcal{R}$ and the minimum cardinality of a right TDS in $G$ is the right domination number, denoted $r(G)$, of $G$. We note that a right TDS of $G$ belongs to $\mathcal{L}$.

\subsection{Preliminary Results}

Cockayne et al. Cockayne et al. (1980) obtained the following upper bound on the total domination number of a connected graph in terms of the order of the graph.

Theorem 3 (Cockayne et al. (1980)) If $G$ is a connected graph of order $n \geq 3$, then $\gamma_{t}(G) \leq 2 n / 3$.

We shall need the following observation.

Observation 4 Let $G_{1} \oplus G_{2}=K(s, s)$, and let $i \in\{1,2\}$. Then the following hold.

(a) $\delta_{i}^{L}+\Delta_{3-i}^{L}=\delta_{i}^{R}+\Delta_{3-i}^{R}=s$.

(b) $\delta_{i}^{L}+\delta_{3-i}^{R} \leq s$.

Proof. Part (a) is immediate. Counting edges in $G_{1}$ and $G_{2}$, we have $m_{i} \geq s \delta_{i}^{L}$ and $m_{3-i} \geq s \delta_{3-i}^{R}$, and so $s^{2}=m_{i}+m_{3-i} \geq s\left(\delta_{i}^{L}+\delta_{3-i}^{R}\right)$, or, equivalently, $\delta_{i}^{L}+\delta_{3-i}^{R} \leq s$.

The following lemma will prove to be useful.

Lemma 5 Let $G_{1} \oplus G_{2}=K(s, s)$, where neither $G_{1}$ nor $G_{2}$ have an isolated vertex, and let $i \in\{1,2\}$. Then the following hold.
(a) $2 \leq \ell_{i}, r_{i} \leq s$.
(b) $\gamma_{t}^{i}=\ell_{i}+r_{i}$.
(c) $\ell_{i} \leq 1+\delta_{3-i}^{R}$ and $r_{i} \leq 1+\delta_{3-i}^{L}$.
(d) $\left(\ell_{i}-2\right)\left(r_{3-i}-1\right) \leq \delta_{3-i}^{R}-1$ and $\left(r_{i}-2\right)\left(\ell_{3-i}-1\right) \leq \delta_{3-i}^{L}-1$.
(e) $\gamma_{t}^{i} \leq 2+2 \min \left\{\Delta_{3-i}^{R}, \Delta_{3-i}^{L}\right\}$. 
Proof. (a) Part (a) follows from the observation that $1 \leq \delta_{i} \leq \Delta_{i} \leq s-1$.

(b) Part (b) follows from the observation that every TDS of $G_{1}$ can be partitioned into two sets, one contained in $\mathcal{L}$ that totally dominates $\mathcal{R}$ and the other contained in $\mathcal{R}$ that totally dominates $\mathcal{L}$.

(c) Let $v \in \mathcal{R}$ be a vertex of degree $\Delta_{1}^{R}$ in $G_{1}$. We now consider the set $N_{2}(v)$ and note that $\left|N_{2}(v)\right|=\delta_{2}^{R}$. For each vertex $u \in N_{2}(v)$, select an arbitrary neighbor $u^{\prime}$ in $G_{1}$. Let $U=\cup\left\{u^{\prime}\right\}$, where the union is taken over all vertices $u \in N_{2}(v)$. Then, $U \cup\{v\}$ is a left TDS in $G_{1}$, and so $\ell_{1} \leq|U|+1 \leq\left|N_{2}(v)\right|+1=1+\delta_{2}^{R}$. The other results follow by symmetry.

(d) Let $v \in \mathcal{R}$ have degree $\Delta_{1}^{R}$ in $G_{1}$ and let $X=\mathcal{L} \backslash N_{1}(v)$. We now partition $X$ into subsets $X_{1}, \ldots, X_{k}$ of size at most $r_{2}-1$ such that $k$ is as small as possible. For each $i=1, \ldots, k$, the set $X_{i}$ does not dominate $\mathcal{R}$ in $G_{2}$, and so there exists a $y_{i} \in \mathcal{R} \backslash N_{2}\left(X_{i}\right)$ which therefore totally dominates $X_{i}$ in $G_{1}$. Hence, $\left\{v, y_{1}, \ldots, y_{k}\right\}$ totally dominates $\mathcal{L}$ in $G_{1}$. Since $k=\left\lceil|X| /\left(r_{2}-1\right)\right\rceil$ and $|X|=s-\Delta_{1}^{R}=\delta_{2}^{R}$, we deduce that $\ell_{1} \leq 1+\left\lceil\delta_{2}^{R} /\left(r_{2}-1\right)\right\rceil \leq 2+\left(\delta_{2}^{R}-1\right) /\left(r_{2}-1\right)$, or, equivalently, $\left(\ell_{1}-2\right)\left(r_{2}-1\right) \leq \delta_{2}^{R}-1$. The other results follow by symmetry.

(e) Let $v \in \mathcal{R}$ have degree $\Delta_{1}^{R}$ in $G_{1}$. Let $X=N_{1}(v)$, and let $u \in \mathcal{L} \backslash X$. Further let $Y=N_{1}(u)$ and $Z=$ $\mathcal{R} \backslash(Y \cup\{v\})$. If some vertex $y \in Y$ dominates $X$ in $G_{1}$, then the degree of $y$ in $G_{1}$ exceeds that of $v$, a contradiction. Hence every $y \in Y$ is adjacent to at least one vertex of $X$ in $G_{2}$, and so $X$ totally dominates $Y$ in $G_{2}$. For each vertex $x \in X$, select an arbitrary neighbor $x^{\prime}$ in $G_{2}$. Let $X^{\prime}=\cup_{x \in X}\left\{x^{\prime}\right\}$ and note that $X^{\prime}$ totally dominates $X$ in $G_{2}$ and $\left|X^{\prime}\right| \leq|X|$. Moreover, $\{u, v\}$ totally dominates $V \backslash(X \cup Y)$ in $G_{2}$. Thus, $X \cup X^{\prime} \cup\{u, v\}$ is a TDS in $G_{2}$, and so $\gamma_{t}^{2} \leq 2+2|X|=2+2 \Delta_{1}^{R}$. Similarly by choosing $v \in \mathcal{L}$ to have degree $\Delta_{1}^{L}$ in $G_{1}$, we have that $\gamma_{t}^{2} \leq 2+2 \Delta_{1}^{L}$. Thus, $\gamma_{t}^{2} \leq 2+2 \min \left\{\Delta_{1}^{R}, \Delta_{1}^{L}\right\}$. Similarly, $\gamma_{t}^{1} \leq 2+2 \min \left\{\Delta_{2}^{R}, \Delta_{2}^{L}\right\}$.

\subsection{The Sum}

In this section, we look at $G_{1} \oplus G_{2}=K(s, s)$ and the upper bound on $\gamma_{t}\left(G_{1}\right)+\gamma_{t}\left(G_{2}\right)$, where neither $G_{1}$ nor $G_{2}$ have an isolated vertex. We shall establish:

Theorem 6 If $G_{1} \oplus G_{2}=K(s, s)$ where neither $G_{1}$ nor $G_{2}$ has an isolated vertex, then $\gamma_{t}\left(G_{1}\right)+$ $\gamma_{t}\left(G_{2}\right) \leq 2 s+4$, with equality if and only if $G_{1}=s K_{2}$ or $G_{2}=s K_{2}$.

Proof. That the upper bound of $2 s+4$ on the sum $\gamma_{t}^{1}+\gamma_{t}^{2}$ is sharp, may be seen by taking $G_{1}=s K_{2}$, in which case $\gamma_{t}^{1}=2 s$ and $\gamma_{t}^{2}=4$, or, by symmetry, taking $G_{2}=s K_{2}$.

We now prove the upper bound holds. Renaming sets, if necessary, we may assume without loss of generality that $r_{1}=\min \left\{\ell_{1}, r_{1}, \ell_{2}, r_{2}\right\}$. By Lemma 5 (a), $r_{1} \geq 2$. If $r_{1}=2$, then we have that

$$
\begin{aligned}
\gamma_{t}^{1}+\gamma_{t}^{2} & =r_{1}+\ell_{1}+\gamma_{t}^{2} & & (\text { by Lemma } 5(\mathrm{~b})) \\
& \leq 2+\left(1+\delta_{2}^{R}\right)+\left(2+2 \Delta_{1}^{R}\right) & & (\text { by Lemma } 5(\mathrm{c}) \text { and } 5(\mathrm{e})) \\
& =5+s+\Delta_{1}^{R} & & (\text { by Observation } 4) \\
& \leq 2 s+4 & & \left(\text { since } \Delta_{1}^{R} \leq s-1\right) .
\end{aligned}
$$

Suppose, further, that $\gamma_{t}^{1}+\gamma_{t}^{2}=2 s+4$ (and still $r_{1}=2$ ). Then equality occurs throughout the above inequality chain. In particular, this requires that $\gamma_{t}^{2}=2+2 \Delta_{1}^{R}$ and that $\Delta_{1}^{R}=s-1$. But then $\gamma_{t}^{2}=2 s$. This is only possible if $G_{2}=s K_{2}$.

Hence we may assume that $r_{1} \geq 3$, for otherwise the desired result follows. By Lemma 5 (c), $r_{1} \leq$ $1+\delta_{2}^{L}$, implying that $\delta_{2}^{L} \geq 2$. Similarly, since $\ell_{1} \geq r_{1} \geq 3$, we note that $\delta_{2}^{R} \geq 2$. Since $r_{2} \geq r_{1} \geq 3$, 
Lemma 5(d) implies that $\ell_{1} \leq 2+\left(\delta_{2}^{R}-1\right) /\left(r_{2}-1\right) \leq 2+\left(\delta_{2}^{R}-1\right) / 2$. Similarly, $r_{1} \leq 2+\left(\delta_{2}^{L}-\right.$ $1) /\left(\ell_{2}-1\right) \leq 2+\left(\delta_{2}^{L}-1\right) / 2$. By Lemma 5 (b), $\gamma_{t}^{1}=\ell_{1}+r_{1}$, and so

$$
\gamma_{t}^{1} \leq 3+\left(\delta_{2}^{R}+\delta_{2}^{L}\right) / 2 .
$$

By Lemma 5.e), $\gamma_{t}^{2} \leq 2+2 \min \left\{\Delta_{1}^{R}, \Delta_{1}^{L}\right\} \leq 2+\Delta_{1}^{R}+\Delta_{1}^{L}$. By Observation 4, we note that $\Delta_{1}^{R}=s-\delta_{2}^{R}$ and $\Delta_{1}^{L}=s-\delta_{2}^{L}$. Hence,

$$
\gamma_{t}^{2} \leq 2+2 s-\delta_{2}^{R}-\delta_{2}^{L}
$$

Adding Equation (1) and Equation 2), we have that $\gamma_{t}^{1}+\gamma_{t}^{2} \leq 2 s+5-\left(\delta_{2}^{R}+\delta_{2}^{L}\right) / 2$. However as observed earlier, $r_{1} \geq 3$ implies that $\delta_{2}^{L} \geq 2$ and $\delta_{2}^{R} \geq 2$. Hence, $\gamma_{t}^{1}+\gamma_{t}^{2} \leq 2 s+5-2<2 s+4$.

\subsection{The Product}

In this section, we look at $G_{1} \oplus G_{2}=K(s, s)$ and the upper bound on $\gamma_{t}\left(G_{1}\right) \gamma_{t}\left(G_{2}\right)$, where neither $G_{1}$ nor $G_{2}$ has an isolated vertex. We shall establish:

Theorem 7 If $G_{1} \oplus G_{2}=K(s, s)$ where neither $G_{1}$ nor $G_{2}$ have an isolated vertex, then $\gamma_{t}\left(G_{1}\right) \gamma_{t}\left(G_{2}\right) \leq$ $\max \left\{8 s,\left\lfloor(s+6)^{2} / 4\right\rfloor\right\}$.

Proof. That the upper bound of $8 s$ on the product $\gamma_{t}^{1} \gamma_{t}^{2}$ is achievable, may be seen by taking $G_{1}=s K_{2}$ or $G_{2}=s K_{2}$. We remark that if $G_{1} \cong B_{12}$, where $B_{12}$ is the bipartite cubic graph of order $n=12$ shown in Figure 1 , then $G_{2} \cong B_{12}$ and $\gamma_{t}^{1} \gamma_{t}^{2}=36=\left\lfloor(s+6)^{2} / 4\right\rfloor$. This shows that the upper bound of $\left\lfloor(s+6)^{2} / 4\right\rfloor$ on the product $\gamma_{t}^{1} \gamma_{t}^{2}$ is achievable.

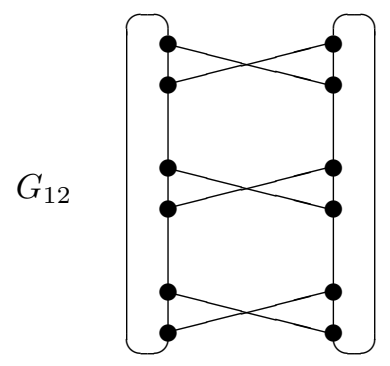

Fig. 1: The bipartite cubic graph $B_{12}$.

If $\gamma_{t}^{1}=4$, then since $\gamma_{t}^{2} \leq 2 s$, we have that $\gamma_{t}^{1} \gamma_{t}^{2} \leq 8 s$. Further if $\gamma_{t}^{1} \gamma_{t}^{2}=8 s$, then $\gamma_{t}^{2}=2 s$ which is only possible if $G_{2}=s K_{2}$. Similarly, if $\gamma_{t}^{2}=4$, then $\gamma_{t}^{1} \gamma_{t}^{2} \leq 8 s$, with equality if and only if $G_{1}=s K_{2}$. Hence we may assume that $\gamma_{t}^{1} \geq 5$ and $\gamma_{t}^{2} \geq 5$, for otherwise the desired result follows. In particular, this implies that both $G_{1}$ and $G_{2}$ are connected. Renaming sets, if necessary, we may assume without loss of generality that $r_{1}=\min \left\{\ell_{1}, r_{1}, \ell_{2}, r_{2}\right\}$. By Lemma 5 (a), $r_{1} \geq 2$.

We proceed further with the following claim.

Claim A: If $r_{1} \geq 3$, then $\gamma_{t}^{1} \gamma_{t}^{2} \leq\left\lfloor(s+6)^{2} / 4\right\rfloor$.

PROOF. An identical argument as in the proof of Theorem 6 , shows that Equation (1) holds; that is, $\gamma_{t}^{1} \leq$ $3+\left(\delta_{2}^{R}+\delta_{2}^{L}\right) / 2$. By a symmetric argument, $\gamma_{t}^{2} \leq 3+\left(\delta_{1}^{R}+\delta_{1}^{L}\right) / 2$. Adding these two inequalities yields $\gamma_{t}^{1}+\gamma_{t}^{2} \leq 6+\left(\delta_{1}^{L}+\delta_{2}^{L}+\delta_{1}^{R}+\delta_{2}^{R}\right) / 2$. Hence, by Observation 4 b), $\gamma_{t}^{1}+\gamma_{t}^{2} \leq s+6$. Since the geometric 
mean is at most the arithmetic mean, we note that $\sqrt{\gamma_{t}^{1} \gamma_{t}^{2}} \leq\left(\gamma_{t}^{1}+\gamma_{t}^{2}\right) / 2$, and so $\gamma_{t}^{1} \gamma_{t}^{2} \leq\left\lfloor(s+6)^{2} / 4\right\rfloor$. This completes the proof of Claim A. (口)

By Claim A we may assume that $r_{1}=2$. We now let $\ell_{1}=k+1$ and continue with the following claim. Claim B: We may assume that the following hold.

(a) $\ell_{2} \leq s-k$.

(b) $4 \leq r_{2}<s / k+1$.

(c) $4 \leq k<s / 3$.

Proof. (a) Let $X$ be a minimum left TDS of $G_{1}$ and note that $X \subseteq \mathcal{R}$ and $|X|=\ell_{1}$. Let $X=$ $\left\{x_{1}, \ldots, x_{\ell_{1}}\right\}$. By the minimality of $X$, every vertex $x \in X$ has at least one neighbor in $G_{1}$ which has no other neighbor in $X$ except for $x$. For each $x_{i} \in X$, let $y_{i}$ be such a neighbor, and so $N_{1}\left(y_{i}\right) \cap X=\left\{x_{i}\right\}$. Let $Y=\left\{y_{1}, \ldots, y_{\ell_{1}}\right\}$ and note that $Y \subseteq \mathcal{L}$ and that $|X|=|Y|=\ell_{1}$. Furthermore, $Y \backslash\left\{y_{i}\right\} \subseteq N_{2}\left(x_{i}\right)$ for all $i \in\left\{1, \ldots, \ell_{1}\right\}$. Let $Y^{\prime}=\mathcal{L} \backslash Y=\left\{y_{1}^{\prime}, \ldots, y_{s-\ell_{1}}^{\prime}\right\}$.

Suppose there are no edges between $X$ and $Y^{\prime}$ in $G_{2}$, that is $G_{2}\left[X, Y^{\prime}\right]=\emptyset$. Since $G_{2}$ is connected, there must exist vertices $y_{j_{1}} \in Y$ and $y_{j_{2}}^{\prime}$ in $Y^{\prime}$ with a common neighbor, $x^{\prime}$ say, in $G_{2}$. Necessarily, $x^{\prime} \in \mathcal{R} \backslash X$. Now, for each $i \in\left\{1, \ldots, s-\ell_{1}\right\} \backslash\left\{j_{2}\right\}$, we let $x_{i}^{\prime} \in N_{2}\left(y_{i}^{\prime}\right)$, and let $x_{j_{2}}^{\prime}=x^{\prime}$. Let

$$
X^{\prime}=\bigcup_{i=1}^{s-\ell_{1}}\left\{x_{i}^{\prime}\right\}
$$

and note that $\left|X^{\prime}\right| \leq s-\ell_{1}$. But now $Y \backslash\left\{y_{j_{1}}\right\} \subseteq N_{2}\left(x_{j_{1}}\right)$ and $Y^{\prime} \cup\left\{y_{j_{1}}\right\} \subseteq N_{2}\left(X^{\prime}\right)$, and so $X^{\prime} \cup\left\{x_{j_{1}}\right\}$ is a left TDS of $G_{2}$. Hence, $\ell_{2} \leq\left|X^{\prime} \cup\left\{x_{j_{1}}\right\}\right| \leq s-\ell_{1}+1=s-k$, as desired.

Hence we may assume that $G_{2}\left[X, Y^{\prime}\right] \neq \emptyset$. Thus for some $k_{1} \in\left\{1, \ldots, \ell_{1}\right\}$ and some $k_{2} \in\{1, \ldots, s-$ $\left.\ell_{1}\right\}$, we have $x_{k_{1}} y_{k_{2}}^{\prime} \in E_{2}$. For each $i \in\left\{1, \ldots, s-\ell_{1}\right\} \backslash\left\{k_{2}\right\}$, we let $x_{i}^{\prime \prime} \in N_{2}\left(y_{i}^{\prime}\right)$, and let $x_{k_{2}}^{\prime \prime}=x_{k_{1}}$. Let

$$
X^{\prime \prime}=\bigcup_{i=1}^{s-\ell_{1}}\left\{x_{i}^{\prime \prime}\right\}
$$

and note that $\left|X^{\prime \prime}\right| \leq s-\ell_{1}$. Let $k_{3} \in\left\{1, \ldots, \ell_{1}\right\} \backslash\left\{k_{1}\right\}$. Then, $Y \backslash\left\{y_{k_{3}}\right\} \subseteq N_{2}\left(x_{k_{3}}\right)$ and $Y^{\prime} \cup\left\{y_{k_{3}}\right\} \subseteq$ $N_{2}\left(X^{\prime \prime}\right)$, and so $X^{\prime \prime} \cup\left\{x_{k_{3}}\right\}$ is a left TDS of $G_{2}$. Hence, $\ell_{2} \leq\left|X^{\prime \prime} \cup\left\{x_{k_{3}}\right\}\right| \leq s-\ell_{1}+1=s-k$, as desired.

(b) Suppose $r_{2} \leq 3$. Recall that $\ell_{1}=k+1, r_{1}=2$, and by part (a), $\ell_{2} \leq s-k$. Therefore, $\gamma_{t}^{1} \gamma_{t}^{2}=$ $\left(\ell_{1}+r_{1}\right)\left(\ell_{2}+r_{2}\right) \leq(k+3)(s-k+3)$. Since the geometric mean is at most the arithmetic mean, we have that $\sqrt{(k+3)(s-k+3)} \leq(s+6) / 2$, and so $\gamma_{t}^{1} \gamma_{t}^{2} \leq\left\lfloor(s+6)^{2} / 4\right\rfloor$. Hence we may assume that $r_{2} \geq 4$. We now partition $\mathcal{R}$ into subsets $X_{1}, \ldots, X_{j}$ of size at most $k$ such that $j$ is as small as possible. Since $\ell_{1}=k+1$, for each $i=1, \ldots, j$, the set $X_{i}$ does not dominate $\mathcal{L}$ in $G_{1}$, and so there exists a $y_{i} \in \mathcal{L} \backslash N_{1}\left(X_{i}\right)$ which therefore totally dominates $X_{i}$ in $G_{2}$. Hence, $\left\{y_{1}, \ldots, y_{j}\right\}$ totally dominates $\mathcal{R}$ in $G_{2}$. Since $j=\lceil|\mathcal{R}| / k\rceil$ and $|\mathcal{R}|=s$, we deduce that $r_{2} \leq\lceil s / k\rceil<s / k+1$.

(c) Suppose $k \leq 3$. Then, $\gamma_{t}^{1}=r_{1}+\ell_{1} \leq 6$. By Theorem $3 . \gamma_{t}^{2} \leq 2\left|V_{2}\right| / 3=4 s / 3$. Hence, $\gamma_{t}^{1} \gamma_{t}^{2} \leq$ $6(4 s / 3)=8 s$. We may therefore assume that $k \geq 4$. By Part (b), we may assume $4<s / k+1$ and thus $k<s / 3$. This completes the proof of Claim B. (a) 
By Claims A and B we now have that $r_{1}=2, \ell_{1}=k+1, r_{2}<s / k+1$, and $\ell_{2} \leq s-k$. Hence, by Lemma 5 (b), we have

$$
\gamma_{t}^{1} \gamma_{t}^{2}<(k+3)(s / k+s-k+1) .
$$

We proceed with the following claim which introduces an assumption we can make on the size of $s$ and improves the bounds in Claim B(b) and Claim B(c).

Claim C: We may assume that the following hold.

(a) $s \geq 20$.

(b) $5 \leq r_{2}<s / k+1$.

(c) $4 \leq k<s / 4$.

Proof. (a) Suppose $s=19$. Then by Claim B(c) we have $k \in\{4,5,6\}$. Substituting these values into Equation (3) we get $\gamma_{t}^{1} \gamma_{t}^{2}<145.25, \gamma_{t}^{1} \gamma_{t}^{2}<150.4$, and $\gamma_{t}^{1} \gamma_{t}^{2}<154.5$, respectively. In each case $\gamma_{t}^{1} \gamma_{t}^{2}<156=\left\lfloor(s+6)^{2} / 4\right\rfloor$, and thus we may assume $s \neq 19$. Suppose now that $s \leq 18$. By Claim B(c) we have $k \in\{4,5\}$. If $k=4$ then

$$
\begin{aligned}
\gamma_{t}^{1} \gamma_{t}^{2} & <(7)(s / 4+s-3) & (\text { substituting into Equation (3) }) \\
& =8 s+3 s / 4-21 & \\
& <8 s & (\text { since } 3 s / 4 \leq 3(18) / 4<21)
\end{aligned}
$$

Hence we may assume that $k=5$. But now we have

$$
\begin{aligned}
\gamma_{t}^{1} \gamma_{t}^{2} & <(8)(s / 5+s-4) & & \text { (substituting into Equation }(3) \\
& <8 s & & \text { (since } s / 5 \leq 18 / 5<4)
\end{aligned}
$$

Hence we may assume that $s \geq 20$.

(b) By Claim B(b) we have $4 \leq r_{2}<s / k+1$. If $r_{2}=4$ then

$$
\begin{array}{rlrl}
\gamma_{t}^{1} \gamma_{t}^{2} & =\left(\ell_{1}+r_{1}\right)\left(\ell_{2}+r_{2}\right) & & (\text { by Lemma } 5(\mathrm{~b})) \\
& \leq(k+3)(s-k+4) & \text { (by Claim B(a)). }
\end{array}
$$

We note that $(k+3)(s-k+4)$ is a parabola as a function of $k$ which achieves its maximum at $k=(s+1) / 2$ and is therefore strictly increasing on the interval $[4, s / 3]$. Therefore, since $4 \leq k<s / 3$ (by Claim $\mathrm{B}(\mathrm{c})$ ), we have

$$
\begin{aligned}
\gamma_{t}^{1} \gamma_{t}^{2} & <(s / 3+3)(s-s / 3+4) \\
& =2 s^{2} / 9+10 s / 3+12 \\
& =(s+6)^{2} / 4-s^{2} / 36+s / 3+3
\end{aligned}
$$

But by Part (a) we have $s>18$, and so $s^{2} / 36>18 s / 36=s / 2=s / 3+s / 6>s / 3+3$. Thus, $-s^{2} / 36+s / 3+3<0$ and the above inequality chain reduces to $\gamma_{t}^{1} \gamma_{t}^{2}<(s+6)^{2} / 4$, and hence $\gamma_{t}^{1} \gamma_{t}^{2} \leq\left\lfloor(s+6)^{2} / 4\right\rfloor$. We may therefore assume that $r_{2} \neq 4$, and so $5 \leq r_{2}<s / k+1$.

(c) By Claim B(c) we have $k \geq 4$. By Part (b), we may assume $5<s / k+1$ and thus $k<s / 4$. This completes the proof of Claim $\mathrm{C}$. ()

We return to the proof of Theorem77 Multiplying out Equation (3) yields

$$
\gamma_{t}^{1} \gamma_{t}^{2}<s+3 s / k+(k+3)(s-k+1) .
$$


We note that since $k \geq 4$ we have that $3 s / k \leq 3 s / 4$. Furthermore, $(k+3)(s-k+1)$ is a parabola as a function of $k$ which achieves its maximum at $k=(s-2) / 2$ and is therefore strictly increasing on the interval [4,s/4]. Using this information and the fact that $4 \leq k<s / 4$ (by Claim C(c)) in Equation (4), we get

$$
\begin{aligned}
\gamma_{t}^{1} \gamma_{t}^{2} & <s+3 s / 4+(s / 4+3)(3 s / 4+1) \\
& =3 s^{2} / 16+17 s / 4+3 \\
& =(s+6)^{2} / 4-s^{2} / 16+5 s / 4-6 .
\end{aligned}
$$

But by Claim C(a) we have $s \geq 20$ and so $s^{2} / 16 \geq 20 s / 16=5 s / 4$. Thus, $-s^{2} / 16+5 s / 4-6<0$ and the above inequality chain reduces to $\gamma_{t}^{1} \gamma_{t}^{2}<(s+6)^{2} / 4$. We conclude that $\gamma_{t}^{1} \gamma_{t}^{2} \leq\left\lfloor(s+6)^{2} / 4\right\rfloor$. This completes the proof of Theorem 7

That the bound of Theorem 7 is essentially best possible, may be seen as follows. For $s \geq 2$, by taking $G_{1}=s K_{2}$ we note that $\gamma_{t}\left(G_{1}\right)=2 s$ and $\gamma_{t}\left(G_{2}\right)=4$, whence $\gamma_{t}\left(G_{1}\right) \gamma_{t}\left(G_{2}\right)=8 s$. Let $s \geq 4$ and let $K(s, s)$ have partite sets $A$ and $B$. Partition $A(B)$ into two sets, one of size $\lfloor s / 2\rfloor$, say $A_{1}\left(B_{1}\right)$, and one of size $\lceil s / 2\rceil$, say $A_{2}\left(B_{2}\right)$. Form the edge set of $G_{1}$ from the set of all edges between $A$ and $B_{1}$ by removing (the edges of) a matching between $A_{1}$ and $B_{1}$ and inserting a matching between $A_{2}$ and $B_{2}$. If $S$ is a TDS of $G_{1}$, then $A_{2} \subset S$ in order to totally dominate $B_{2}$, while $\left|S \cap B_{1}\right| \geq 2$ in order to totally dominate $A_{1}$. Hence, $\gamma_{t}\left(G_{1}\right) \geq\lceil s / 2\rceil+2$. However adding any two vertices of $B_{1}$ to the set $A_{2}$ produces a TDS of $G_{1}$. Consequently, $\gamma_{t}\left(G_{1}\right)=\lceil s / 2\rceil+2$. Similarly, $\gamma_{t}\left(G_{2}\right)=\lfloor s / 2\rfloor+2$. Thus, $\gamma_{t}\left(G_{1}\right) \gamma_{t}\left(G_{2}\right)=(\lceil s / 2\rceil+2)(\lfloor s / 2\rfloor+2)=\left\lfloor(s / 2+2)^{2}\right\rfloor=\left\lfloor(s+4)^{2} / 4\right\rfloor$.

We remark that $\left\lfloor(s+6)^{2} / 4\right\rfloor \geq 8 s$ for $s \geq 18$.

\section{References}

S. Arumugan and A. Thuraiswamy. Total domination in graphs. Ars Combin., 43:89-92, 1996.

G. Chartrand and J. Mitchem. Graphical theorems of the Nordhaus-Gaddum class. In M. Capobianco, J. Frechen, and M. Krolik, editors, Recent Trends in Graph Theory, volume 186 of Lecture Notes in Mathematics, pages 55-61. Springer Berlin / Heidelberg, 1971.

E. J. Cockayne, R. M. Dawes, and S. T. Hedetniemi. Total domination in graphs. Networks, 10(3): 211-219, 1980.

S. Erfang, D. Chuangyin, and K. Liying. A note on Nordhaus-Gaddum inequalities for domination. Discrete Applied Math., 136:83-85, 2004.

O. Favaron, H. Karami, R. Khoeilar, S. M. Sheikholeslami, and L. Volkmann. Proof of a conjecture on game domination. J. Graph Theory, 64:323-329, 2010.

Z. Füredi, A. Kostochka, R. Škrekovski, M. Stiebitz, and D. B. West. Nordhaus-Gaddum-type theorems for decompositions into many parts. J. Graph Theory, 50:273-292, 2005.

W. Goddard and M. A. Henning. Nordhaus-Gaddum bounds for independent domination. Discrete Math., 268:299-302, 2003.

W. Goddard, M. A. Henning, and H. C. Swart. Some Nordhaus-Gaddum-type results. J. Graph Theory, 16:221-231, 1992. 
F. Harary and T. W. Haynes. Nordhaus-Gaddum inequalities for domination in graphs. Discrete Math., (155):99-105, 1996.

J. H. Hattingh, E. Jonck, E. J. Joubert, and A. R. Plummer. Nordhaus-Gaddum results for restrained domination and total restrained domination in graphs. Discrete Math., 308:1080-1087, 2008.

T. Haynes, S. Hedetniemi, and P. Slater. Fundamentals of domination in graphs. Monographs and textbooks in pure and applied mathematics. Marcel Dekker, 1998a.

T. Haynes, S. Hedetniemi, and P. Slater. Domination in graphs: advanced topics. Monographs and textbooks in pure and applied mathematics. Marcel Dekker, Inc., $1998 \mathrm{~b}$.

M. A. Henning. Recent results on total domination in graphs: A survey. Discrete Math., 309:32-63, 2009.

M. A. Henning, E. J. Joubert, and J. Southey. Nordhaus-Gaddum bounds for total domination. Appl. Math. Lett., 24(6):987-990, 2011.

F. Jaeger and C. Payan. Relations du type Nordhaus-Gaddum pour le nombre d'absorption d'un graphe simple. C.R. Acad. Sci. Ser. A, 274:728-730, 1972.

J. P. Joseph and S. Arumugam. Domination in graphs. Internat. J. Management Systems, 11:177-182, 1995.

J. P. k. Bounds on chromatic numbers of multiple factors of a complete graph. J. Graph Theory, 2:9-17, 1978.

E. A. Nordhaus and J. W. Gaddum. On complementary graphs. Amer. Math. Monthly, 63:175-177, 1956.

C. Payan and N. Xuong. Domination-balanced graphs. J. Graph Theory, 6:23-32, 1982. 
